\title{
Metallic Nickel Silicides: Experiments and Theory for NiSi and First Principles Calculations for Other Phases
}

\author{
Ashutosh Dahal $^{\mathrm{a}}$, Jagath Gunasekera ${ }^{\mathrm{a}}$, Leland Harringer ${ }^{\mathrm{b}}$, Deepak K. Singh ${ }^{\mathrm{a}}$, \\ David J. Singh ${ }^{\mathrm{a}}$ \\ ${ }^{a}$ Department of Physics and Astronomy, University of Missouri, Columbia, MO \\ 65211-7010 USA \\ ${ }^{b}$ NIST Center for Neutron Research, Gaithersburg, MD 20899 USA
}

\begin{abstract}
We report detailed experimental investigation of the transport and magnetic properties of orthorhombic NiSi along with first principles studies of this phase and related nickel silicides. Neutron scattering shows no evidence for magnetism, in agreement with first principles calculations. Comparison of first principles results and experimental results from our measurements and literature show a weak electron phonon coupling. We discuss transport and other properties of $\mathrm{NiSi}$ and find behavior characteristic of a weak correlated metal far from magnetism. Trends among the nickel silicides as a function of nickel content are discussed.
\end{abstract}

\section{Introduction}

Nickel silicides are important electronic materials that have been used as contacts for field effect transistors, as interconnects and in nanoelectronic devices. $[1,2,3,4,5,6,7]$ The Ni-Si binary phase diagram shows several ordered compounds all of which are metallic except for the end point, Si. There are both $\mathrm{Si}$ rich and $\mathrm{Ni}$ rich compounds. The compounds include $\mathrm{Ni}_{3} \mathrm{Si}_{1} \mathrm{Ni}_{31} \mathrm{Si}_{12}$, $\mathrm{Ni}_{2} \mathrm{Si}, \mathrm{Ni}_{3} \mathrm{Si}_{2}$, NiSi and $\mathrm{NiSi}_{2}$. Also, although $\mathrm{Ni}$ is an elemental ferromagnet, magnetic order has not been reported in the Ni-Si compounds.

The compounds in the Ni-Si phase diagram have been studied in various levels of detail previously. $[8,9,10,11,12,13,6]$ This includes a study of the energetics of the different phases by first principles calculations with the functional known as PBESOL by Christensen and co-workers, [11] who obtained overbinding of the phases relative to existing thermochemical data, $[14,15]$ as well as investigations of the band structure, electronic density of states and related properties. Prior work has shown all the phases to be metallic and has investigated individual compounds to develop better understanding of the metallic properties, $[16,17]$ particularly emphasizing NiSi where quantum oscillations

Email address: singhdj@missouri.edu (David J. Singh) 
were measured on single crystals. [17] The low resistivities that characterize the metallic state of NiSi also transfer to nanostructures, where values as low as 10 $\mu \Omega \mathrm{cm}$ have been obtained along with extremely high current carrying capacities. [18]

The purpose of this paper is to report in detail the electronic and related properties of these materials as obtained from first principles calculations, along with an experimental investigation of the NiSi phase. This is the most widely used phase in applications. We report structural data consisting of fully relaxed atomic coordinates for all compounds, energetics, that are in much better accord with experiment than prior reports, and a detailed analysis of the electronic structures including plasma frequencies, electronic densities of states, thermopowers and other quantities in relation to experiment. We also report detailed spin-polarized calculations including fixed spin moment calculations showing absence of magnetism. This result is confirmed by low temperature neutron diffraction, which proves that there is no magnetic ordering in NiSi going down to $0.48 \mathrm{~K}$. Comparison of experimental and theoretical results shows that NiSi has weak electron phonon scattering. The results show behavior consistent with a metallic compound far from magnetic instabilities and in a weakly correlated regime. This provides an explanation for the good metallic conductivity. The first principles results suggest similar behavior for the other metallic nickel silicides, although there is an interesting Fermi surface structure with a low dimensional sheet in cubic $\mathrm{Ni}_{3} \mathrm{Si}$.

\section{Experimental Methods}

The high purity polycrystalline samples of NiSi were synthesized by conventional solid state reaction method using ultra-pure ingredients (99.9\% purity powders obtained from Alfa Aesar) of Ni and Si. Starting materials were mixed in stoichiometric composition, pelletized and sintered for twenty four hours. We obtained high quality samples for sintering temperatures of $950^{\circ} \mathrm{C}$ and $970^{\circ} \mathrm{C}$. The sample for which data is given was prepared by (1) ramping to $970^{\circ} \mathrm{C}$ over four hours, (2) holding at $970^{\circ} \mathrm{C}$ for 24 hours and (3) cool down to room temperature over eight hours. This sintering was done under vacuum in a sealed quartz tube. The furnace used was a rapid temperature 1700 series front loader manufactured by CM Inc. The resulting samples were characterized using a Siemens D500 powder X-ray diffractometer. The X-ray diffraction (XRD) data was refined using the Reitveld powder diffraction refinement. As shown in Fig. 1 , every single peak of the XRD pattern was identified with the Pnma orthorhombic structure of NiSi, with lattice parameters of $a=5.186 \AA, b=3.331$ Åand $c=5.625 \AA$. The XRD pattern and the lattice parameters are consistent with other reports on the synthesis of high quality samples of NiSi. The refined structural parameters are $x_{\mathrm{Ni}}=0.0090, z_{\mathrm{Ni}}=0.1854, x_{\mathrm{Si}}=0.1790$ and $z_{\mathrm{Si}}=0.5874$. These are in good accord with prior data and our first principles calculated values (see below). The electrical properties of $\mathrm{NiSi}$ was characterized using the four probe technique and utilizing a closed-cycle refrigerator cooled $9 \mathrm{~T}$ magnet 


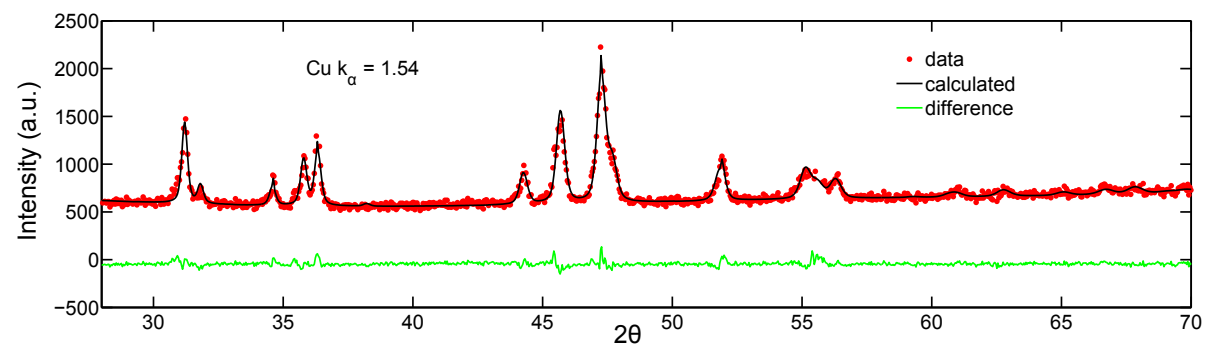

Figure 1: Powder X-ray diffraction spectra of NiSi powder, used in this study. The powder diffraction data is refined using FullProf suite for Rietveld analysis. The high purity of the sample is evident from the XRD data, where every single peak is identified to the orthorhombic structure (crystallographic group Pnma) of NiSi.

with measurement temperature range of 1.5-300 K. Elastic neutron scattering measurements were performed on the $3.6 \mathrm{~g}$ pristine powder sample of NiSi on the spin-polarized triple-axis spectrometer (SPINS) at the NIST Center for Neutron Research with fixed final neutron energy of $5 \mathrm{meV}$. The measurements employed a flat pyrolytic graphite (PG) analyzer with collimator sequence of Mono- $80^{\prime}$-sample-Be filter- $80^{\prime}-3$ blades flat analyzer- $120^{\prime}$ - detector.

\section{Computational Methods}

The present calculations were performed within density functional theory using the generalized gradient approximation of Perdew, Burke and Ernzerhof (PBE-GGA), [19] and the general potential linearized augmented planewave (LAPW) method [20] as implemented in the WIEN2k code. [21] This is an all electron method in which both core and valence states are treated selfconsistently. LAPW sphere radii of $2.25 \mathrm{bohr}$ and $1.85 \mathrm{bohr}$ were used for $\mathrm{Ni}$ and $\mathrm{Si}$, respectively, along with well converged basis sets of the standard LAPW plus local orbital type, with local orbitals for the Ni p semi-core state and a planewave cutoff $k_{\max }$ corresponding to $R_{\min } k_{\max }=7.5$, where $R_{\min }=1.85$ bohr is the Si radius (for an effective Ni value in excess of 9). Dense Brillouin zone samplings were used and convergence with respect to zone sampling was checked. Transport functions were calculated with the BoltzTraP code [22] and plasma frequencies were calculated using the optical package of WIEN2k. In all cases we used the experimental lattice parameters and determined any free internal coordinates by total energy minimization. These calculated internal coordinates are given in Table 1, along with the lattice parameters used. For high symmetry phases, we used $\mathrm{Ni}_{3} \mathrm{Si}$ : cubic $(P m \overline{3} m)$, lattice parameter $a=3.5098$ $\AA$, and $\mathrm{NiSi}_{2}$ : cubic $(F m \overline{3} m), a=5.406 \AA$. $[23,24,25]$

\section{Experimental Results and Discussion}

As mentioned, NiSi is a low resistance compensated metal.[17] Previous electrical and Hall probe measurements, especially on the single crystal specimen, 


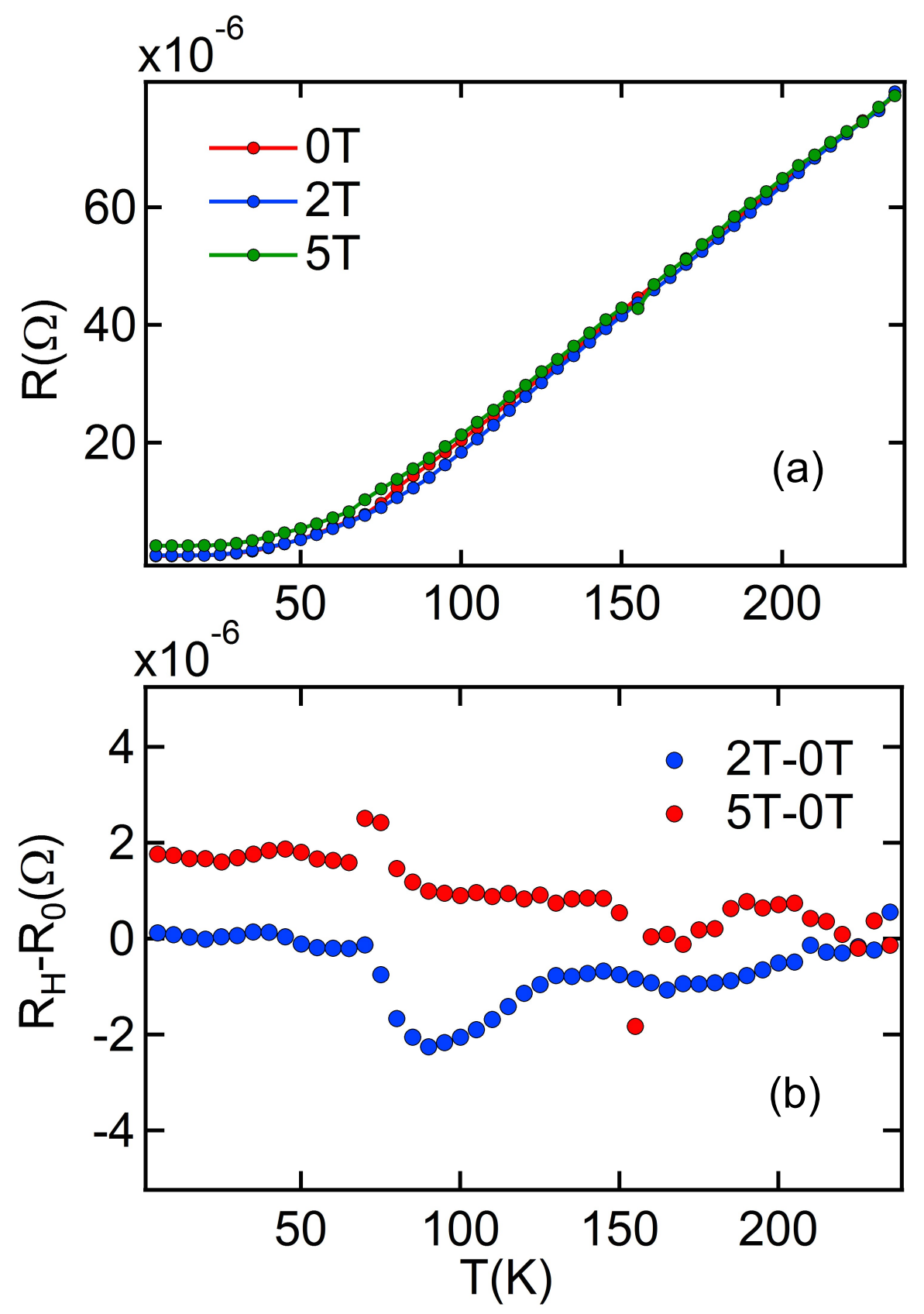

Figure 2: Electrical resistance of NiSi. (a) Electrical resistance as a function of temperature in applied field of $H=0 \mathrm{~T}, 2 \mathrm{~T}$ and $5 \mathrm{~T}$. (b) $\Delta R=R(\mathrm{H})-R(0)$ as a function of temperature. 
Table 1: Calculated internal structural parameters in lattice units with the literature experimental lattice parameters for $\mathrm{NiSi}(a=5.1818 \AA, b=3.334 \AA ⿻$, $c=5.619 \AA$, space group 62 , Pnma), $\mathrm{Ni}_{3} \mathrm{Si}_{2}(a=12.229 \AA, b=10.805 \AA$, $c=6.924 \AA$, space group $36, C m c 21), \mathrm{Ni}_{2} \mathrm{Si}(a=4.992$ $\AA, b=3.741 \AA, c=7.061 \AA$, space group $62, P n m a), \mathrm{Ni}_{31} \mathrm{Si}_{12}(a=b=6.671 \AA, c=12.288 \AA$, space group 150, P321).

\begin{tabular}{|c|c|c|c|}
\hline & $x$ & $y$ & $z$ \\
\hline \multicolumn{4}{|l|}{ NiSi: } \\
\hline $\mathrm{Ni} 4 c$ & 0.0083 & 0.2500 & 0.1880 \\
\hline Si $4 c$ & 0.1789 & 0.2500 & 0.5826 \\
\hline \multicolumn{4}{|l|}{$\mathbf{N i}_{3} \mathbf{S i}_{2}:$} \\
\hline Ni1 $8 b$ & 0.1717 & 0.1189 & 0.1193 \\
\hline $\mathrm{Ni} 28 b$ & 0.3028 & 0.2533 & 0.3086 \\
\hline $\mathrm{Ni3} 8 b$ & 0.3191 & 0.0046 & 0.3068 \\
\hline $\mathrm{Ni} 48 b$ & 0.3276 & 0.3813 & 0.9978 \\
\hline $\mathrm{Ni} 54 a$ & 0.0000 & 0.9999 & 0.0574 \\
\hline $\mathrm{Ni} 64 a$ & 0.0000 & 0.2343 & 0.6199 \\
\hline $\mathrm{Ni} 74 a$ & 0.0000 & 0.2347 & 0.9964 \\
\hline $\mathrm{Ni} 84 a$ & 0.0000 & 0.3830 & 0.3095 \\
\hline Si1 $4 a$ & 0.0000 & 0.5924 & 0.3075 \\
\hline $\mathrm{Si} 28 b$ & 0.1517 & 0.3415 & 0.1004 \\
\hline $\mathrm{Si} 38 b$ & 0.3482 & 0.1573 & 0.0177 \\
\hline $\mathrm{Si} 48 b$ & 0.3808 & 0.4431 & 0.3081 \\
\hline $\mathrm{Si} 54 a$ & 0.0000 & 0.1562 & 0.3082 \\
\hline \multicolumn{4}{|l|}{$\mathrm{Ni}_{2} \mathrm{Si}:$} \\
\hline Ni1 $4 c$ & 0.0412 & 0.2500 & 0.7057 \\
\hline $\mathrm{Ni} 24 c$ & 0.1705 & 0.2500 & 0.0602 \\
\hline Si $4 c$ & 0.2122 & 0.2500 & 0.3860 \\
\hline \multicolumn{4}{|c|}{$\mathbf{N i}_{31} \mathbf{S i}_{12}$ : } \\
\hline Ni1 $6 g$ & 0.0341 & 0.3573 & 0.1948 \\
\hline $\mathrm{Ni} 26 g$ & 0.0786 & 0.4120 & 0.4045 \\
\hline $\mathrm{Ni} 36 g$ & 0.3348 & 0.2978 & 0.3022 \\
\hline $\mathrm{Ni} 46 g$ & 0.3703 & 0.3083 & 0.0963 \\
\hline $\mathrm{Ni} 52 d$ & $1 / 3$ & $2 / 3$ & 0.0671 \\
\hline Ni6 $2 d$ & $1 / 3$ & $2 / 3$ & 0.5728 \\
\hline $\mathrm{Ni} 72 c$ & 0.0000 & 0.0000 & 0.4029 \\
\hline $\mathrm{Ni} 81 a$ & 0.0000 & 0.0000 & 0.0000 \\
\hline Si1 $3 f$ & 0.6815 & 0.0000 & 0.5000 \\
\hline $\mathrm{Si} 22 d$ & $1 / 3$ & $2 / 3$ & 0.7755 \\
\hline $\mathrm{Si} 33 e$ & 0.3510 & 0.0000 & 0.0000 \\
\hline $\mathrm{Si} 42 d$ & $1 / 3$ & $2 / 3$ & 0.2736 \\
\hline $\mathrm{Si} 52 c$ & 0.0000 & 0.0000 & 0.2073 \\
\hline
\end{tabular}




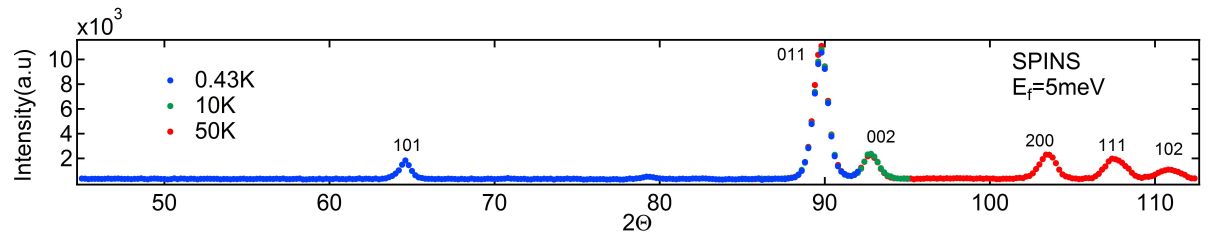

Figure 3: Neutron scattering investigation of possible magnetic order in NiSi. Observed sharp peaks in this figure are attributed to the nuclear structure factor. The difference between the elastic scans data at $0.48 \mathrm{~K}$ and $50 \mathrm{~K}$ is found to be statistically insignificant. Hence, no evidence of any magnetic order is detected. The error bars represent one standard deviation.

of NiSi revealed two interesting properties: (a) very small resistivity of $\sim 10$ $\mu \Omega \mathrm{cm}$ at room temperature, and (b) the change in the nature of the charge carrier at $T \simeq 40 \mathrm{~K}$. The sign of Hall coefficient, $R_{H}$, changes from negative, at $T \geq 40 \mathrm{~K}$, to positive at $T \leq 40 \mathrm{~K}$.[16] This is not unusual in metals and also frequently occurs in compensated semimetals. NiSi is a compensated (even number of electrons) metal. We performed electrical measurements in both zero and applied magnetic field on the high quality polycrystalline sample of NiSi. Characteristic plots of electrical resistance versus temperature in applied fields of $H=0,2$ and $5 \mathrm{~T}$ are shown in Fig. 2a (our sample had a complex shape and so we do not report resistivity) It is noticed that the electrical resistance in applied fields does not exhibit any significant departure from the zero field curve as a function of temperature.

Further quantitative information is obtained by plotting the difference between the zero field and the applied field resistance data, $\Delta R=R(\mathrm{H})-R(0)$, as a function of temperature. As shown in Fig. 2b, $\Delta R$ fluctuates between positive and negative values at $H=2 \mathrm{~T}$. Although the net change in the electrical resistance at this field is small compared to the bulk value at $H=0 \mathrm{~T}$, the fluctuation is more prominent between $70 \mathrm{~K}$ and $100 \mathrm{~K}$. Unlike the electrical behavior in $2 \mathrm{~T}$ field, $\Delta R$ remains positive at all temperatures in $5 \mathrm{~T}$ field.

Next, we discuss the magnetic properties of $\mathrm{NiSi}$. We have performed elastic neutron scattering measurements at low temperature up to $T=0.48 \mathrm{~K}$. Elastic neutron data at few characteristic temperatures are plotted in Fig. 3. As shown in this figure, no additional peaks are detected beyond the lattice Bragg peaks, signifying the orthorhombic structure of the system. Moreover, the relative intensities of the Bragg peaks do not exhibit any temperature dependence as the difference between the peak intensities is statistically insignificant. This rules out any ferromagnetism in the system. No magnetic order is detected in NiSi to the lowest measurement temperature of $T=0.48 \mathrm{~K}$. Neutron diffraction going to very low temperature is fully consistent with the known crystal structure and with x-ray diffraction at high temperature. This means that there are no peaks from magnetic ordering, which excludes any antiferromagnetic ordering. Since the material is not ferromagnetic or ferrimagnetic this means that NiSi has no magnetic ordering. 


\section{First Principles Results and Discussion}

The calculated energetics are summarized in Fig. 4. All the phases are either on the convex hull or very close to it. All of these nickel silicide compounds are found to be metallic. Finally the binding energies of the compounds relative to the end points are high reaching $0.537 \mathrm{eV} /$ atom at the bottom of the convex hull. This indicates that the compounds are very stable and that unreacted end point elements are highly unlikely in the NiSi samples. The calculated values deviate from reported experimental data by $\sim 0.05 \mathrm{eV} /$ atom with a systematic overbinding of the compounds relative to that data. $[14,15]$ This may be within the experimental uncertainty, which has been estimated to be $0.04 \mathrm{eV} /$ atom for $\mathrm{NiSi}_{2}$. [26] This overbinding is much smaller than the overbinding reported in prior calculations [11] that used the PBESOL functional. [27] PBESOL is a functional designed to improve calculated lattice parameters at the expense of binding energies. We verified that the functional is the origin of the difference by repeating the calculations with the PBESOL functional for $\mathrm{Ni}_{3} \mathrm{Si}$ and $\mathrm{NiSi}_{2}$, for which we obtained an increase in the overbinding by $0.07 \mathrm{eV} /$ atom and 0.11 $\mathrm{eV} /$ atom, respectively. Interestingly, $\mathrm{Ni}_{31} \mathrm{Si}_{12}$ and $\mathrm{Ni}_{3} \mathrm{Si}_{2}$ are slightly above the convex hull, but are on it to the precision expected in density functional calculations. The phase diagram clearly shows a $\mathrm{Ni}_{3} \mathrm{Si}_{2}$ phase. However, $\mathrm{Ni}_{31} \mathrm{Si}_{12}$ is a difficult to stabilize compound, perhaps consistent with an energy above the convex hull.

Table 2 gives properties of the metallic state in the various compounds. Interestingly, the density of states at the Fermi level, $N\left(E_{F}\right)$, varies by less than a factor of two between the difference silicide compounds, but the plasma energies, $\Omega_{p}=\hbar \omega_{p}$, show much larger differences as well significant but still relatively modest anisotropy in certain compounds, specifically $\mathrm{Ni}_{3} \mathrm{Si}_{2}$ and to a lesser extent NiSi. In metals the conductivity $\sigma=\omega_{p}^{2} \tau$, where $\tau$ is an effective inverse scattering rate that is both temperature and material dependent. The inverse scattering rate in this formula generally has weak direction dependence relative to the plasma energy in three dimensionally bonded materials. In this case the conductivity anisotropy has weak temperature dependence and is given by the anisotropy of $\Omega_{p}^{2}$. In NiSi this amounts a maximum anisotropy of $\sigma_{x x} / \sigma_{z z}=1.47$, while in $\mathrm{Ni}_{3} \mathrm{Si}_{2}, \sigma_{y y} / \sigma_{x x}=2.34$.

Importantly, based on the plasma energies, $\mathrm{NiSi}, \mathrm{NiSi}_{2}$ and to a lesser extent $\mathrm{Ni}_{2} \mathrm{Si}$ are expected to be high conductivity materials, while $\mathrm{Ni}_{3} \mathrm{Si}, \mathrm{Ni}_{31} \mathrm{Si}_{12}$ and $\mathrm{Ni}_{3} \mathrm{Si}_{2}$ are expected to be lower conductivity materials. This is potentially important because $\mathrm{Ni}_{3} \mathrm{Si}_{2}$ is the neighboring phase to $\mathrm{NiSi}$ in the phase diagram. As such $\mathrm{Ni}$ rich NiSi material may contain $\mathrm{Ni}_{3} \mathrm{Si}_{2}$, i.e. a lower conductivity minority phase that may have a disproportionate effect on electrical transport. On the other hand $\mathrm{Si}$ rich material would contain $\mathrm{NiSi}_{2}$, which is expected to be a higher conductivity material and have less effect on the transport.

The last column of Table 2 gives the ceramic average values of the Seebeck coefficient at $300 \mathrm{~K}$. These values were calculated from $S_{a v}=\left(\sigma_{x x} S_{y y}+\sigma_{y y} S_{z z}+\right.$

$\left.\sigma_{z z} S_{z z}\right) /\left(\sigma_{x x}+\sigma_{y y}+\sigma_{z z}\right)$, replacing $\sigma$ by $\Omega_{p}^{2}$, i.e. using an isotropic relaxation time. The values of $S_{a v}$ are relatively low, consistent with the metallic nature 


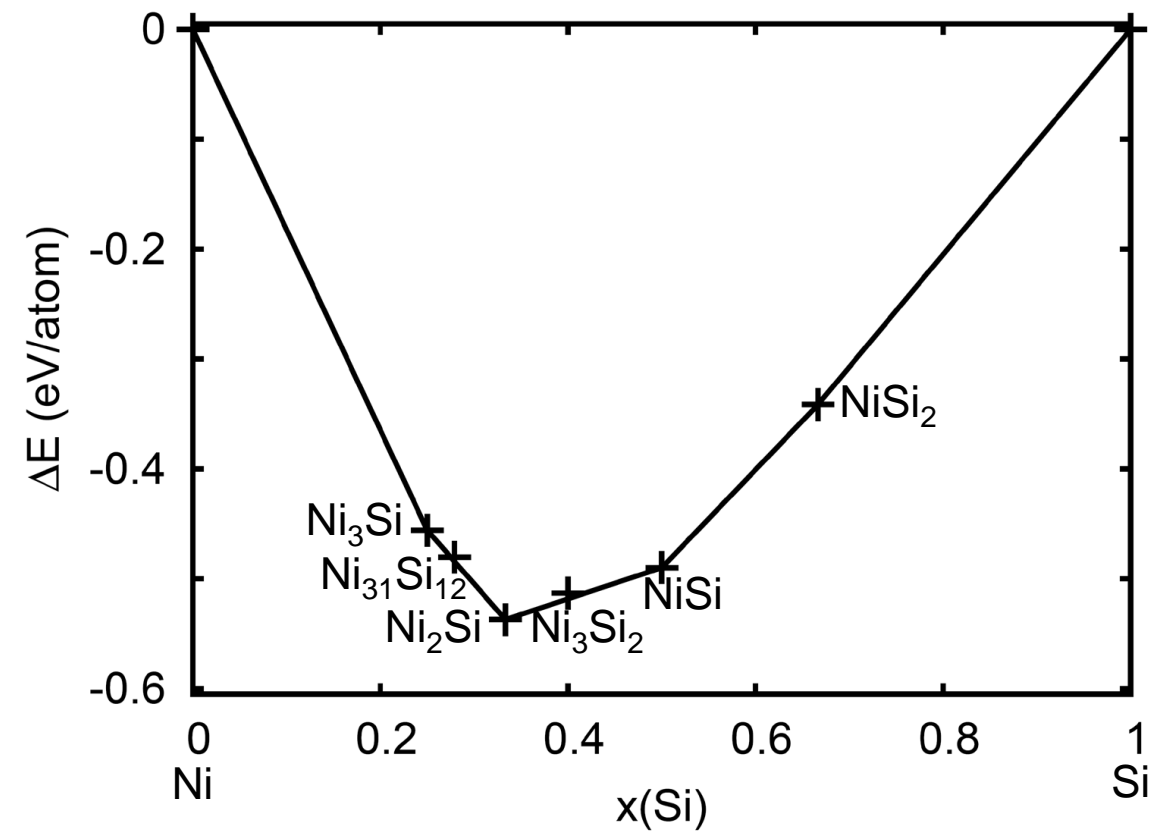

Figure 4: Convex hull with calculated energies of Ni-Si compounds relative to a mixture of the end point compounds on a per atom basis. The end points are ferromagnetic fcc $\mathrm{Ni}$ and diamond structure Si.

of these compounds. At $300 \mathrm{~K}$ they are weakly negative (n-type) for all the compounds except $\mathrm{Ni}_{3} \mathrm{Si}$.

The Fermi surfaces of these compounds are generally complex, large and for some sheets open, reflecting the metallic nature of the materials. Fig. 5 shows the Fermi surface of $\mathrm{NiSi}$, which is the main subject of this paper, as well as that of $\mathrm{Ni}_{3} \mathrm{Si}$, which shows an interesting two dimensional nature for one of the sheets even though the material is cubic. Such features have been discussed in the context of thermoelectric materials, [28] although $\mathrm{Ni}_{3} \mathrm{Si}$ is clearly has far to low of a Seebeck coefficient to be an interesting thermoelectric. The corresponding band structure of $\mathrm{NiSi}$, which is similar to that reported previously by Connetable and Thomas, $[9,8]$ and that of $\mathrm{Ni}_{3} \mathrm{Si}$ are shown in Figs. 6 and 7 , respectively. The open sheets of Fermi surface in particular lead to strong anisotropy in the thermopowers, normally the most isotropic transport property in semiconductors. For NiSi, the calculated direction dependent thermopowers at $300 \mathrm{~K}$ are $S_{x x}=-6.0 \mu \mathrm{V} / \mathrm{K}, S_{y y}=-1.5 \mu \mathrm{V} / \mathrm{K}$ and $S_{z z}=+0.1 \mu \mathrm{V} / \mathrm{K}$, where $x$, $y$ and $z$ are along the crystallographic $a, b$ and $c$ directions. The corresponding densities of states are given in Figs. 8 and 9, respectively.

The density of states at the Fermi level, $N\left(E_{F}\right)$ for NiSi corresponds to a bare electronic specific heat coefficient, $\gamma_{\text {bare }}=1.29 \mathrm{~mJ} /\left(\mathrm{mol} \mathrm{K}^{2}\right)$. The measured value from literature [16] is $\gamma=1.73 \mathrm{~mJ} /\left(\mathrm{mol} \mathrm{K}^{2}\right)$. This yields an inferred 


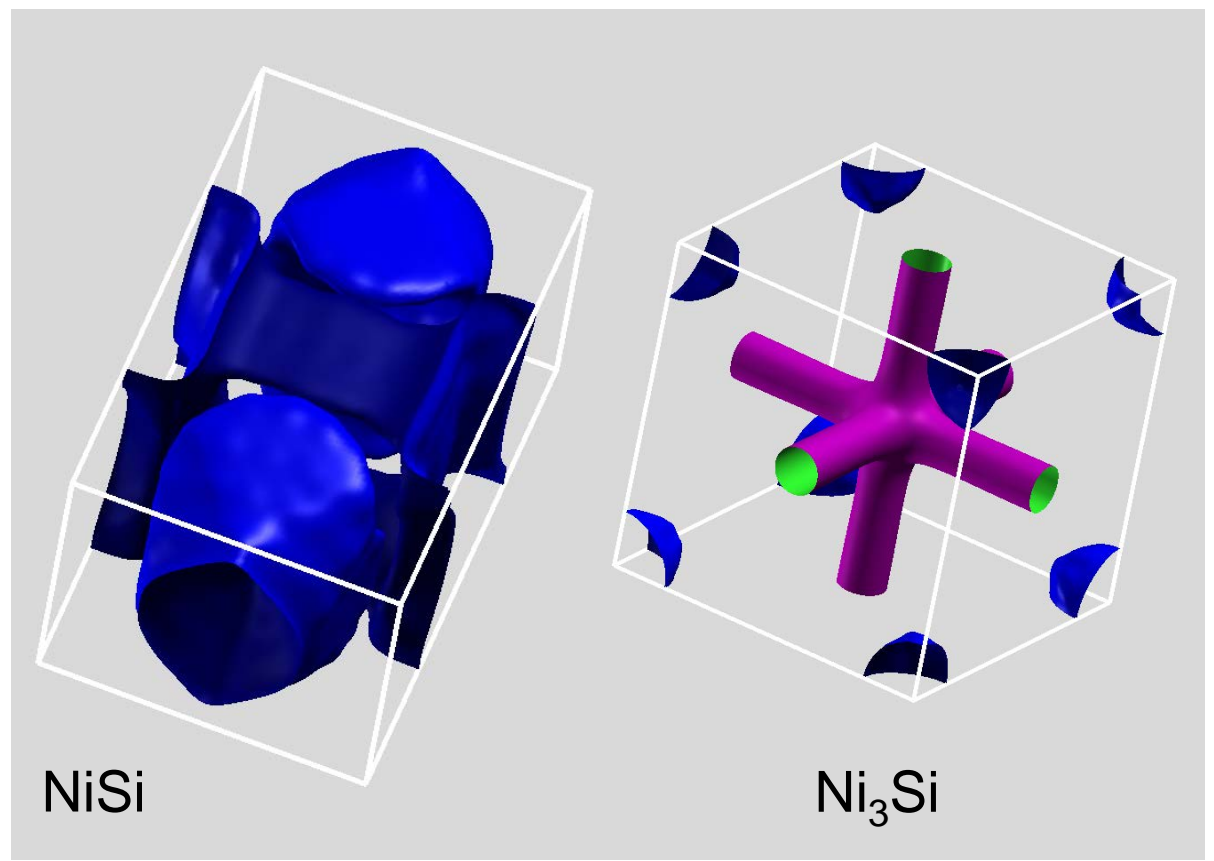

Figure 5: Calculated Fermi surfaces of orthorhombic NiSi and cubic $\mathrm{Ni}_{3} \mathrm{Si}$. The Brillouin zones are given by the white lines.

Table 2: Properties of the metallic state in nickel silicides. The density of states at the Fermi level $N\left(E_{F}\right)$ is given in $e V^{-1}$ on a per nickel atom basis, plasma energies $\Omega_{p}$ in eV and $300 \mathrm{~K}$ ceramic average thermopowers, $S_{a v}(300 K)$ in $\mu \mathrm{V} / \mathrm{K}$.

\begin{tabular}{lccccc}
\hline & $N\left(E_{F}\right)$ & $\Omega_{p, x x}$ & $\Omega_{p, y y}$ & $\Omega_{p, z z}$ & $S_{a v}(300 K)$ \\
\hline $\mathrm{Ni}_{3} \mathrm{Si}$ & 0.83 & 1.77 & 1.77 & 1.77 & 1 \\
$\mathrm{Ni}_{31} \mathrm{Si}_{12}$ & 0.84 & 2.02 & 2.02 & 1.68 & -7 \\
$\mathrm{Ni}_{2} \mathrm{Si}$ & 0.83 & 4.25 & 4.42 & 4.00 & -15 \\
$\mathrm{Ni}_{3} \mathrm{Si}_{2}$ & 0.51 & 1.60 & 2.45 & 2.32 & -12 \\
$\mathrm{NiSi}$ & 0.55 & 5.76 & 5.44 & 4.77 & -3 \\
$\mathrm{NiSi}_{2}$ & 0.86 & 5.57 & 5.57 & 5.57 & -4 \\
\hline
\end{tabular}




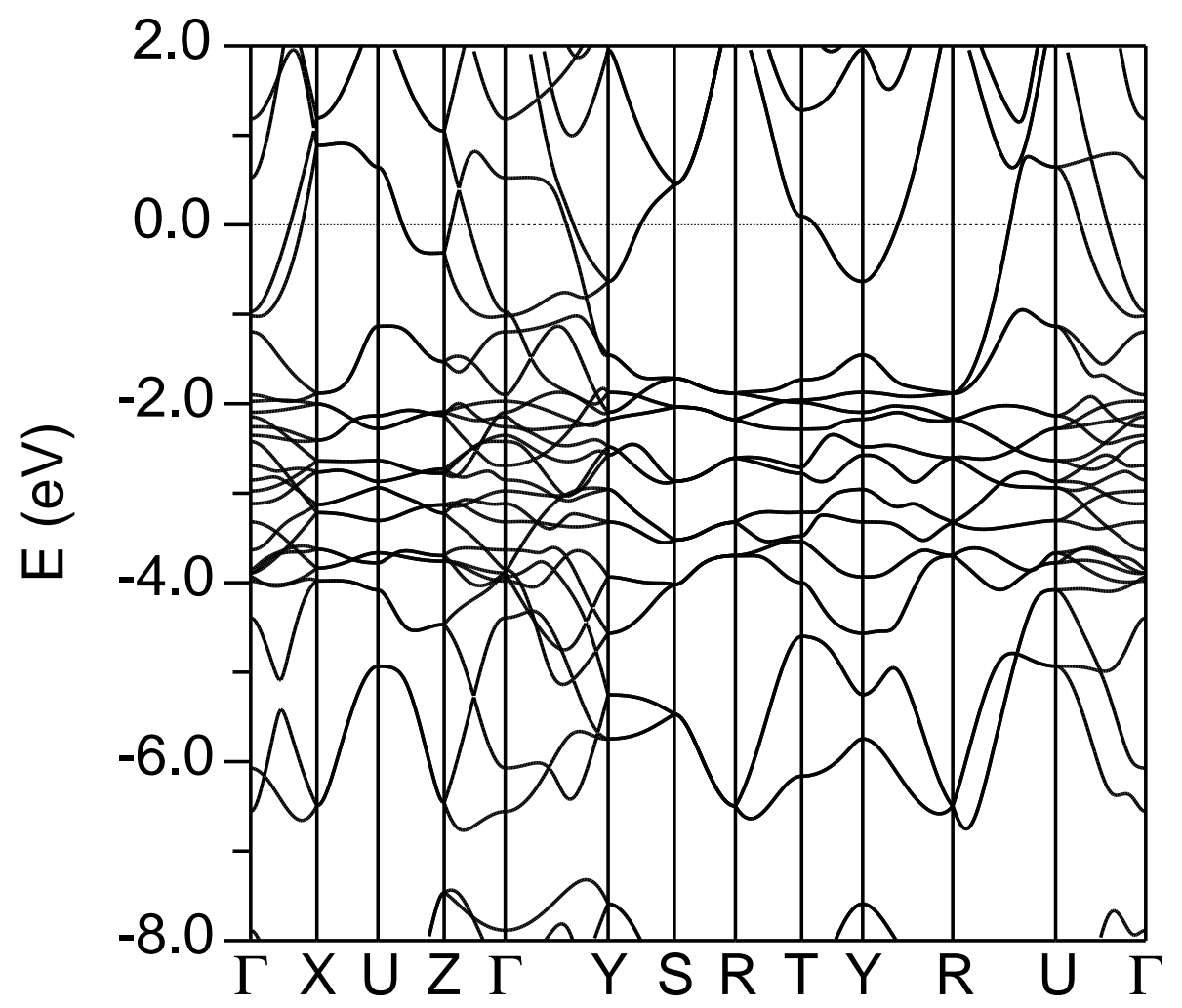

Figure 6: Calculated band structure of orthorhombic NiSi.

enhancement, $\gamma / \gamma_{\text {bare }}=(1+\lambda)=1.34$, i.e. a modest specific heat $\lambda=0.34$.

As mentioned, within Boltzmann theory the resistivity and plasma frequency are related. In particular, the resistivity, $\rho^{e p}$ that comes from electron phonon scattering can be written

$$
\rho^{e p}=\frac{1}{4 \pi}\left(\Omega_{p}^{2} \tau_{e p}\right)^{-1},
$$

where at high $T$

$$
\tau_{e p}^{-1}=\left(2 \pi \lambda_{t r} k_{B} T / \hbar\right) .
$$

Here $\lambda_{t r}$ is an electron phonon coupling constant related to the $\lambda$ that governs electron phonon superconductivity. [29, 30] Our experimental results for the resistance $R(T)$ of NiSi show a long near linear region from $\sim 75 \mathrm{~K}$ to at least $300 \mathrm{~K}$, consistent with prior single crystal data. [16] Meyer and co-workers reported an absolute $\rho_{x x}=10 \mu \Omega \mathrm{cm}$ for current along the crystallographic $a$ axis, which is the high conductivity axis according to the anisotropy of the calculated plasma frequencies. Poon and co-workers obtained a value of $\rho=15 \mu \Omega \mathrm{cm}$ for a film on polycrystalline Si. [31] We estimate a slope $d \rho / d T=3.6 \times 10^{-10} \Omega \mathrm{m} / \mathrm{K}$ 


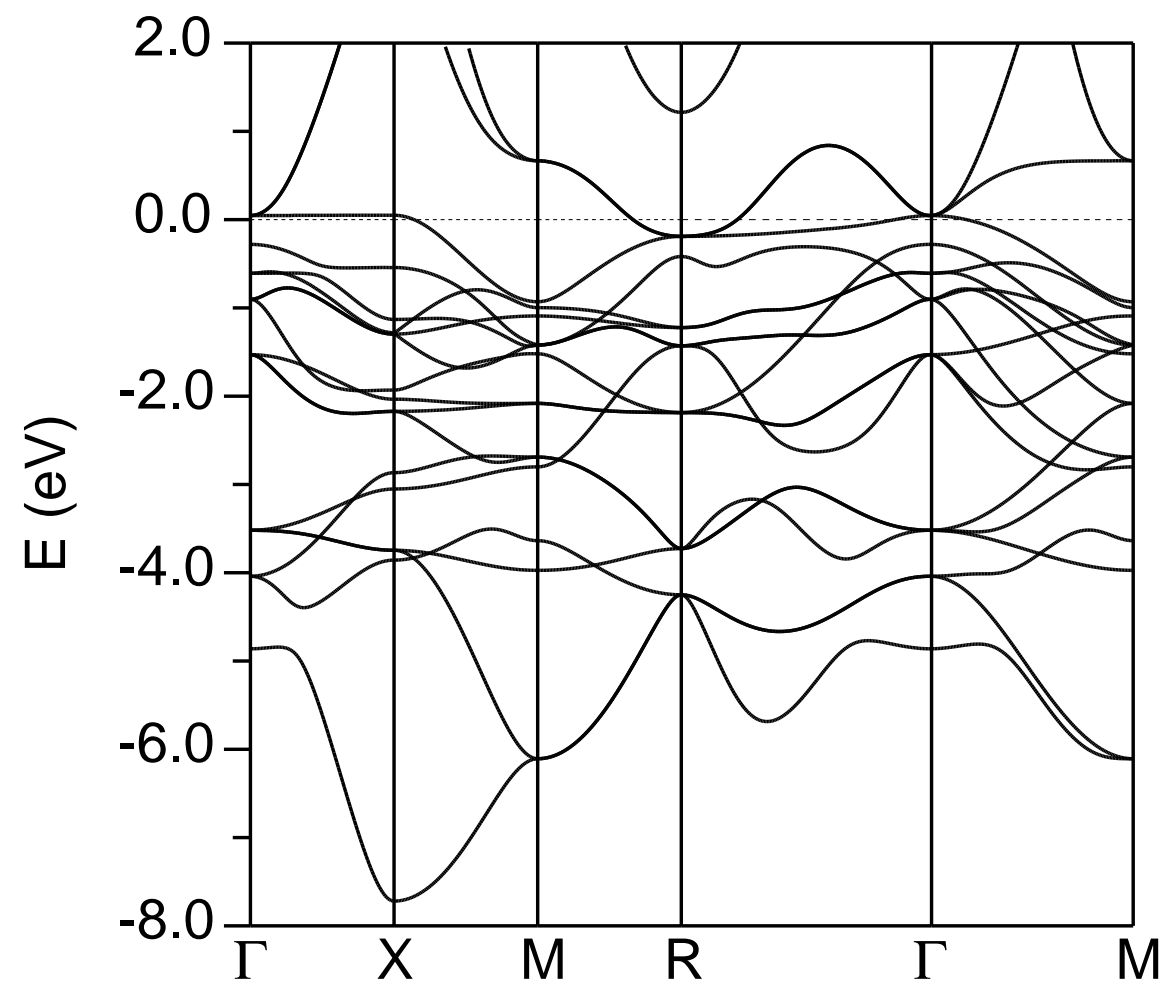

Figure 7: Calculated band structure of cubic $\mathrm{Ni}_{3} \mathrm{Si}$. 


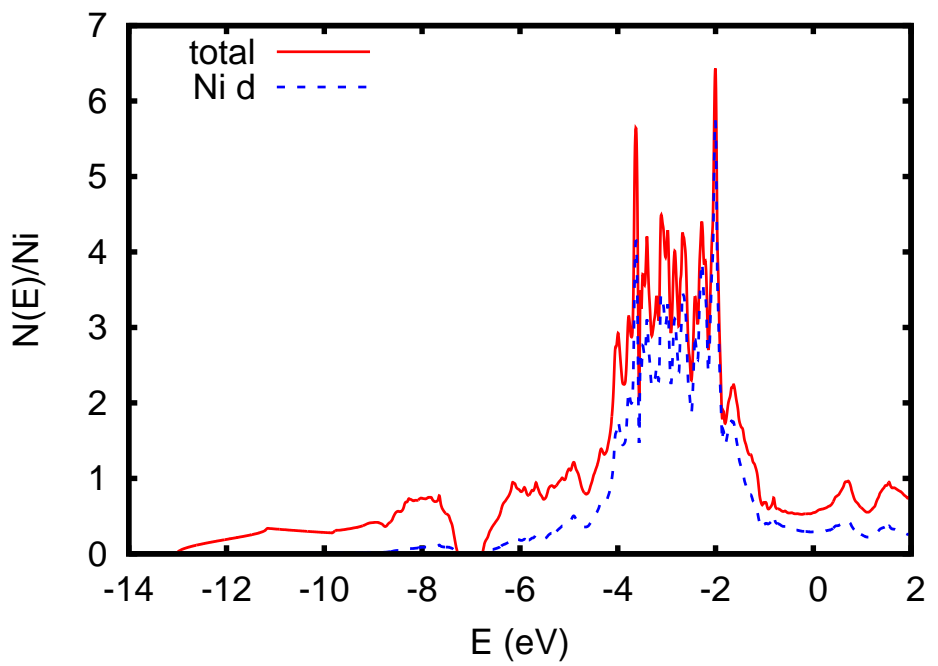

Figure 8: Calculated electronic density of states and $\mathrm{Ni} d$ contribution on a per $\mathrm{Ni}$ atom basis for NiSi.

by combining the value $\rho_{x x}=10 \mu \Omega \mathrm{cm}$ at $300 \mathrm{~K}$ with our measured resistivity. Taking the plasma energy, $\Omega_{p, x x}=5.76 \mathrm{eV}$, we obtain an estimated $\lambda_{t r}=0.30$, which is consistent with the specific heat enhancement. Therefore based on specific heat and resistivity in combination with the present band structure results we infer an electron phonon coupling constant $\lambda \sim 0.3$.

This value suggests that if there are no spin-fluctuations due to proximity to magnetism or magnetic impurities, NiSi could be a low temperature superconductor. This is based on normal values of the Coulomb repulsion parameter $\mu^{*} \sim 0.12-0.15$ in the Allen-Dynes equation, [32]

$$
T_{c}=\frac{\omega_{e f f}}{1.2} \exp \left(\frac{-1.04(1+\lambda)}{\lambda-\mu^{*}(1+0.62 \lambda)}\right),
$$

where $\omega_{\text {eff }}$ is an average phonon frequency for the spectral function. In any case, while the approximation of setting $\lambda$ for this equation equal to $\lambda_{t r}$ or the $\lambda$ from the specific heat enhancement is clearly rough, the results suggest that high quality NiSi samples that do not have magnetic impurities may exhibit superconductivity. This would be at very low temperature. With reasonable values of $\mu^{*}$ and $\omega_{\text {eff }}$ one obtains $T_{c}$ in the $1-100 \mathrm{mK}$ range due to the weak coupling.

The electronic structures show Fermi levels that are at or above the top of the main Ni $d$ bands (see Figs. 8 and 9), similar to previous density functional 


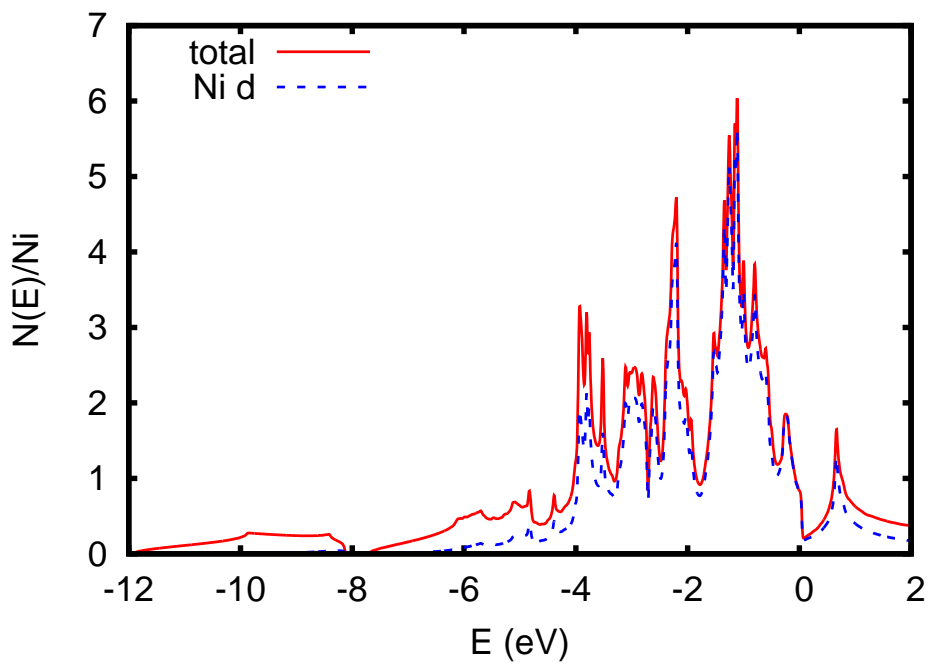

Figure 9: Calculated electronic density of states and $\mathrm{Ni} d$ contribution on a per $\mathrm{Ni}$ atom basis for $\mathrm{Ni}_{3} \mathrm{Si}$.

calculations for these materials. $[8,9,10,11,12,13,6]$ The results show the trend noted previously that the Ni $d$ bands move below the Fermi level to higher binding energy as the $\mathrm{Si}$ content is increased. This is important in the selection of $\mathrm{Ni}-\mathrm{Si}$ contacts for devices as it controls the level positions, and is in accord with experimental data. $[13,33,34]$

As mentioned, calculated values of $N\left(E_{F}\right)$ are remarkably similar between the different compounds, varying from $0.51 \mathrm{eV}^{-1}\left(\mathrm{Ni}_{3} \mathrm{Si}_{2}\right)$ to $0.86 \mathrm{eV}^{-1}$, when expressed on a per $\mathrm{Ni}$ basis. These values are much lower than the value for pure $\mathrm{Ni}$, which is $N\left(E_{F}\right)=4.9 \mathrm{eV}^{-1}$ for calculations without ferromagnetism (i.e. non-spin-polarized calculations). Within Stoner theory the proximity to itinerant ferromagnetism of a metal is governed by a random phase approximation type expression, $\chi=\chi_{0}\left[1-N\left(E_{F}\right) I\right]^{-1}$, where the Stoner parameter, $I$ is approximately $1 \mathrm{eV}$ for late $3 \mathrm{~d}$ transition elements, such as $\mathrm{Ni}$, and $N\left(E_{F}\right)$ is the density of states, expressed on a per atom, per spin basis (i.e. half of the both spins $N\left(E_{F}\right)$ values given in Table 2). [35, 36, 37]

In practice, $I$ is a material dependent parameter that is reduced by hybridization between transition metal and ligand orbitals, but nonetheless application of the Stoner formula with replacement of $N\left(E_{F}\right)$ by the projection of $N\left(E_{F}\right)$ onto the transition metal orbitals is useful in assessing the proximity of a given metal to a ferromagnetic instability. The instability occurs when $N\left(E_{F}\right)=I^{-1}$, i.e. when the $\mathrm{Ni}$ projection of the density of states, $N_{N i}\left(E_{F}\right)=1 \mathrm{eV}^{-1}$ per spin 


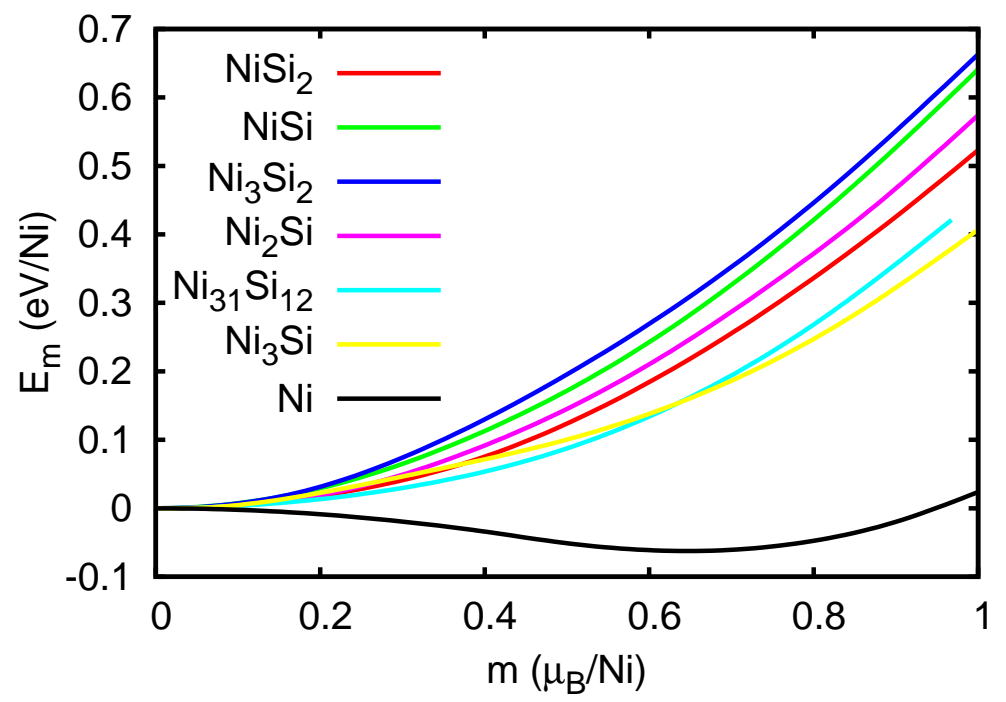

Figure 10: Calculated magnetic energy as a function of constrained spin moment from fixed spin moment calculations. The results are given per Ni atom, both for the magnetic energy and the moment. Elemental fcc $\mathrm{Ni}$ is included for comparison. Note the ferromagnetic instability for the element in contrast to the silicides. 
per atom. Clearly, none of the silicide phases studied is near ferromagnetism by this criterion, and furthermore none of them has a substantial enhancement of their paramagnetism, governed by $\left[1-N\left(E_{F}\right) I\right]^{-1}$.

We did fixed spin moment calculations to verify this. The results of such spin-polarized calculations for all compounds except $\mathrm{Ni}_{31} \mathrm{Si}_{12}$ are shown in Fig. 10. As shown, none of the compounds has a ferromagnetic tendency and the energies as functions of the imposed spin moment are featureless, as is often the case for simple metals that are not near magnetism. We also performed calculations for some simple antiferromagnetic configurations in $\mathrm{NiSi}$, but did not find any stable solutions. These spin polarized calculations, both for initial ferromagnetic and initial antiferromagnetic configurations always converged to zero spin density and an energy equal to that of the non-spin-polarized case. The facts that the energies of non-spin-polarized and spin-polarized calculations are the same and that the self-consistent moment in the cell is zero confirm that there is no magnetism, consistent with the fixed spin moment calculations. This is consistent with our neutron scattering results. Spin polarized calculations for initial ferromagnetic configurations converged to the non-spin-polarized solution for all compounds studied, except pure $\mathrm{Ni}$ for which a ferromagnetic solution is found as expected. The calculated spin moment of fcc Ni is $0.635 \mu_{B}$ per atom.

Within standard Kohn-Sham density functional theory the exchange correlation potential depends on the density and the spin-density. When the spindensity is zero, i.e. when spin-polarized calculations converge to a non-magnetic state, as is the case here, the exchange-correlation potential becomes exactly the same for spin-up and spin-down. Then the electronic density of states is exactly the same for spin up and spin down. Therefore the spin-up and spin-down densities of states are identical and equal to half of the total density of states.

\section{Summary and Conclusions}

We report experimental and theoretical study of NiSi and results of first principles calculations for other nickel silicides, all of which are metallic. Neutron scattering measurements to $0.48 \mathrm{~K}$ confirm that there is no magnetic ordering in NiSi. Combination of first principles results with experimental data show both weak electron phonon scattering and behavior consistent with a metallic compound far from magnetic instabilities and in a weakly correlated regime. The first principles results suggest similar behavior for the other metallic nickel silicides, although there is an interesting Fermi surface structure with a low dimensional sheet in cubic $\mathrm{Ni}_{3} \mathrm{Si}$.

\section{Acknowledgements}

Theoretical work was supported by the Department of Energy, Office of Science, Basic Energy Sciences, through the Computational Synthesis of Materials Software Project. 


\section{References}

[1] E. Hokelek, G. Y. Robinson, Aluminum/nickel silicide contacts on silicon, Thin Solid Films 53 (1978) 135.

[2] Y. Hu, S. P. Tay, Spectroscopic ellipsometry investigation of nickel silicide formation by rapid thermal process, J. Vac. Sci. Tech. A 16 (1998) 1820.

[3] C. Lavoie, F. M. d'Heurle, C. Detavernier, C. Cabral, Towards implementation of a nickel silicide process for cmos technologies, Microelec. Eng. 70 (2003) 144-157.

[4] L. Dong, J. Bush, V. Chirayos, R. Solanki, J. Jiao, Y. Ono, J. F. Conley, Jr., B. D. Ulrich, Dielectrophoretically controlled fabrication of single-crystal nickel silicide interconnects, Nanolett. 5 (2005) 2112-2115.

[5] W. M. Weber, L. Geelhaar, A. P. Graham, E. Unger, G. S. Guesberg, M. L. an W Pamler, C. C. an H Riechert, P. Lugll, F. Kreupl, Silicon-nanowire transistors with intruded nickel-silicide contacts, Nano Lett. 6 (2006) 26602666.

[6] K. T. Butler, J. H. Harding, A computational investigation of nickel silicides as potential contact layers for silicon photovoltaic cells, J. Phys. Condens. Matter 25 (2013) 395003.

[7] J. P. Gambino, E. G. Colgan, Silicides and ohmic contacts, Mater. Chem. Phys. 52 (1998) 99-146.

[8] D. Connetable, O. Thomas, First-principles study of nickel-silicides ordered phases, J. Alloys Compd. 509 (2011) 2639-2644.

[9] D. Connetable, O. Thomas, First-principles study of the structural, vibrational and elastic properties of orthorhombic NiSi, Phys. Rev. B 79 (2009) 094101.

[10] M. Taguchi, F. Le Normand, J. Hommet, S. Rey, G. Schmerber, J. C. Parlebas, Evolution of the electronic structure of NixSiy ordered systems: experimental and theoretical investigations, Eur. Phys. J. B 18 (2000) 611615.

[11] M. Christensen, V. Eyert, C. Freeman, E. Wimmer, A. Jain, J. Blatchford, D. Riley, J. Shaw, Formation of nickel-platinum silicides on a silicon substrate: structure, phase stability and diffusion from ab initio computations, J. Appl. Phys. 114 (2013) 033533.

[12] J. Teyssier, R. Viennois, J. Salamin, E. Giannini, D. van der Marel, Experimental and first principle calculation of $\mathrm{Co}_{x} \mathrm{Ni}_{(1-x)} \mathrm{Si}$ solid solution structural stability, J. Alloys Compd. 465 (2008) 462-467.

[13] A. Franciosi, J. H. Weaver, F. A. Schmidt, Electronic structure of nickel silicides $\mathrm{Ni}_{2} \mathrm{Si}, \mathrm{NiSi}$ and $\mathrm{NiSi}_{2}$, Phys. Rev. B 26 (1982) 546. 
[14] W. Oelsen, H. von. Samson-Himmelstjerna, Mitt. Kaiser-Wilhelm Inst. Eisenforch. Dusseldorf 18 (1936) 131-133.

[15] W. Oelsen, W. Middel, Mitt. Kaiser-Wilhelm Inst. Eisenforch. Dusseldorf 19 (1937) $1-26$.

[16] B. Meyer, U. Gottlieb, O. Laborde, H. Yang, J. C. Lasjaunias, A. Sulpice, R. Madar, Intrinsic properties of NiSi, J. Alloys Compd. 262-263 (1997) 225-237.

[17] R. M. Boulet, A. E. Dunsworth, J. P. Jan, H. L. Skriver, De haas-van alphen effect the lmto bandstructure of NiSi, J. Phys. F 10 (1980) 2197.

[18] Y. Wu, J. Xiang, C. Yang, W. Lu, C. M. Lieber, Single-crystal metallic nanowires and metal/semiconductor nanowire heterostructures, Nature (London) 61 (2004) 61-65.

[19] J. P. Perdew, K. Burke, M. Ernzerhof, Generalized gradient approximation made simple, Phys. Rev. Lett. 77 (18) (1996) 3865-3868.

[20] D. J. Singh, L. Nordstrom, Planewaves Pseudopotentials and the LAPW Method, 2nd Edition, Springer, Berlin, 2006.

[21] P. Blaha, K. Schwarz, G. Madsen, D. Kvasnicka, J. Luitz, WIEN2k, An Augmented Plane Wave + Local Orbitals Program for Calculating Crystal Properties, K. Schwarz, Tech. Univ. Wien, Austria, 2001.

[22] G. K. H. Madsen, D. J. Singh, BoltzTraP a code for calculating bandstructure dependent quantities, Comput. Phys. Commun. 175 (2006) 6771 .

[23] F. Weitzer, J. C. Schuster, Phase diagrams of the ternary systems Mn, Fe, Co, Ni-Si-N, J. Solid State Chem. 70 (1987) 178-184.

[24] K. W. Richter, H. Ipser, The Al-Ni-Si phase diagram between 0 and 33.3 at.\% Ni, Intermetallics 11 (2003) 101-109.

[25] K. W. Richter, K. Chandrasekaran, H. Ipser, The Al-Ni-Si phase diagram. part ii: phase equilibria between 33.3 and 66.7 at.\% Ni, Intermetallics 12 (2004) 545-554.

[26] M. S. Chandrasekharaiah, J. L. Margrave, P. A. G. O'Hare, The discilicides of tungsten, molybdenum, tantalum, titanium, cobalt and nickel and platinum monosilicide: a survey of their thermodynamic properties, J. Phys. Chem. Ref. Data 22 (1993) 1459-1468.

[27] J. P. Perdew, A. Ruzsinszky, G. I. Csonka, O. A. Vydrov, G. E. Scuseria, L. A. Constantin, X. Zhou, K. Burke, Restoring the density-gradientexpansion for exchange in solids and surfaces, Phys. Rev. Lett. 100 (2008) 136406. 
[28] D. Parker, X. Chen, D. J. Singh, High three-dimensional thermoelectric performance from low-dimensional bands, Phys. Rev. Lett. 110 (2013) 146601.

[29] P. B. Allen, Empirical electron-phonon $\lambda$ values from resistivity of cubic metallic elements, Phys. Rev. B 36 (1987) 2920-2923.

[30] P. B. Allen, W. E. Pickett, H. Krakauer, Anisotropic normal-state transport properties predicted and analyszed for high- $T_{c}$ oxide superconductors, Phys. Rev. B 37 (1988) 7482-7490.

[31] M. C. Poon, F. Deng, M. Chan, W. Y. Chan, S. S. Lau, Resistivity and thermal stability of nickel mono-silicide, Appl. Surf. Sci. 157 (2000) 29-34.

[32] P. B. Allen, R. C. Dynes, Transition temperature of strong-coupled superconductors reanalyzed, Phys. Rev. B 12 (1975) 905-922.

[33] A. Humbert, A. Cros, Reflectivity and electronic structures of Ni-silicides, J. Phys. (Paris) 44 (1983) L929-L933.

[34] E. Verleysen, H. Bender, O. Richard, D. Schryvers, W. Vandervorst, Characterization of nickel silicides using eels-based methods, J. Microscopy 240 (2010) 75-82.

[35] E. C. Stoner, Collective electron ferromagnetism II: energy and specific heat, Proc. R. Soc. London Ser. A 169 (1939) 339-371.

[36] O. Gunnarsson, Band model for magnetism of transition metals in the spin-density-functional formalism, J. Phys. F 6 (1976) 587-606.

[37] J. F. Janak, Uniform susceptibilities of metallic elements, Phys. Rev. B 16 (1977) 255-262. 